\title{
Intelligent Fault Diagnosis Model for Rotating Machinery Based on Fusion of Sound Signals
}

\author{
M. Saimurugan ${ }^{1}$, R. Nithesh ${ }^{2}$ \\ ${ }^{1,2}$ Department of Mechanical Engineering, Amrita School of Engineering, Coimbatore, Amrita Vishwa Vidyapeetham, Amrita University, \\ Coimbatore -641112, Tamil Nadu, India. \\ m_saimurugan@cb.amrita.edu \\ nitheshr2014@gmail.com
}

\begin{abstract}
The failure of rotating machine elements causes unnecessary downtime of the machine. Fault in the rotating machinery can be identified from noises, vibration signals obtained from sensors. Bearing and shaft are the most important basic rotating machine elements. Detection of fault from vibration signals is widely used method in condition monitoring techniques for diagnosis of machine elements. Fault diagnosis from sound signals is cost effective than vibration signals. Sound signal analysis is not well explored in the field of automated fault diagnosis. Under various simulated fault conditions, the sound signals are obtained by placing microphone near the bearing for different speeds. The features are extracted by using statistical and histogram methods. The best features of sound signals are obtained by decision tree algorithm. The extracted features are used as inputs to the classifier-Artificial Neural Network. The classification accuracy results from statistical and histogram features are obtained and compared.
\end{abstract}

\section{INTRODUCTION}

Condition monitoring and diagnostic maintenance is gaining its importance in last few decades. Many industries are checking for highly durable and reliable machines that can be efficient and productive for long time. Major causes for failure in machine elements is due to improper design, material and manufacturing defects, installation errors etc. Failure is machinery specific and can be dangerous, so there is a need for scheduled maintenance. Various condition monitoring techniques widely used are vibration signals, sound signals, acoustic emissions, infrared thermography, wear debris analysis etc. The rotating machine elements includes shaft, bearing, gears, pulleys etc. For rotating machine elements vibration signals analysis is widely used. It is important to detect and find fault in the rotating

M. Saimurugan et al, This is an open-access article distributed under the terms of the Creative Commons Attribution 3.0 International License, which permits unrestricted use, distribution, and reproduction in any medium, provided the original author and source are credited. machineries at proper time in order to avoid catastrophic failure of the machine (Xin Lei \& Peter A. Sandborn, 2016). Fault in the different machine elements can be found by signals from the machine using transducers (Robert Bond Randall, 2011). In order to ensure safety, sensors are attached to the components and monitored continuously. An air crash was caused due to bearing failure, as the cage of bearing that supports balls was broken is a catastrophic type (Smalley, Baldwin, Mauney \& Millwater, 1996).

Generally, bearing faults includes cracks on the races, deformation of balls, broken cages. Among these, cracks on the races is frequently occurring fault. So far vibration analysis were used to identify fault in bearing. The sound signals can also be used to find fault in the rolling element bearing. The sound signals are extracted through statistical methods, because these signals are of random or stochastic type can be solved by mathematical expressions and this method gives simple and quick computation results (Heng \& Nor, 1998).

Data mining approach using a machine learning technique is widely used now-a-days because from large sets of data, conclusions can be drawn in an effective manner. Artificial Intelligent techniques such as Artificial Neural Networks (ANN), which resembles same working as that of neural system of human beings based on facts and this method finds symptoms based on the causes (Vyas \& Satishkumar, 2001) and Support Vector Machines (SVM), which forms the advanced method of identification and classification of data based on experience rather than facts (Widodo \& Yang, 2007; Yuan \& Chu, 2007) have been used as classifiers in the fault diagnosis of machines. Most of the research in machine fault detection is done with the help of vibration signals (Kankar, Satish Sharma \& Harsha, 2011). The sound signals are employed in order to reduce the cost of maintenance, so this can be used by small scale industries (Amarnath, Sugumaran $\&$ Hemantha Kumar, 2013). The fusion of signals involves spectral segmentation process. The frequency spectrum of signals are divided initially to fine segments and then merged based on different subsets (Chuan Li, Ming Liang \& Tianyang Wang, 2015). The Artificial Neural Network has an important objective to minimize errors. This method involves of adjustment of weight function based on the training values 
(Samanta \& Al-Balushi, 2003; Alaa Abdulhady Jaber \& Robert Bicker, 2016). The ANN is non-linear model of solving problem. Artificial Neural Network model can easily give origination and severity of faults. The dynamic systems exhibits quasi-periodic, chaotic signals that contains valuable parameter information can be classified efficiently by ANN (Samadani, Kitio Kwuimy \& Nataraj, 2015). Multi-layer neural networks is employed in case of fault detection of gear box using vibration signals and found to provide highly reliable results (Zhiqiang Chen, Chuan Li \& René Vinicio Sánchez, 2015). The vibration signal measurement on an inclined surface requires accelerometer on a Precision tilt table for accurate reading (Meirovitch, 1986). The sound signal analyses involving microphone can be easily fitted at any angle and at any place of the machine. The methodology of the present study is given in the form of flow chart in Figure 1.

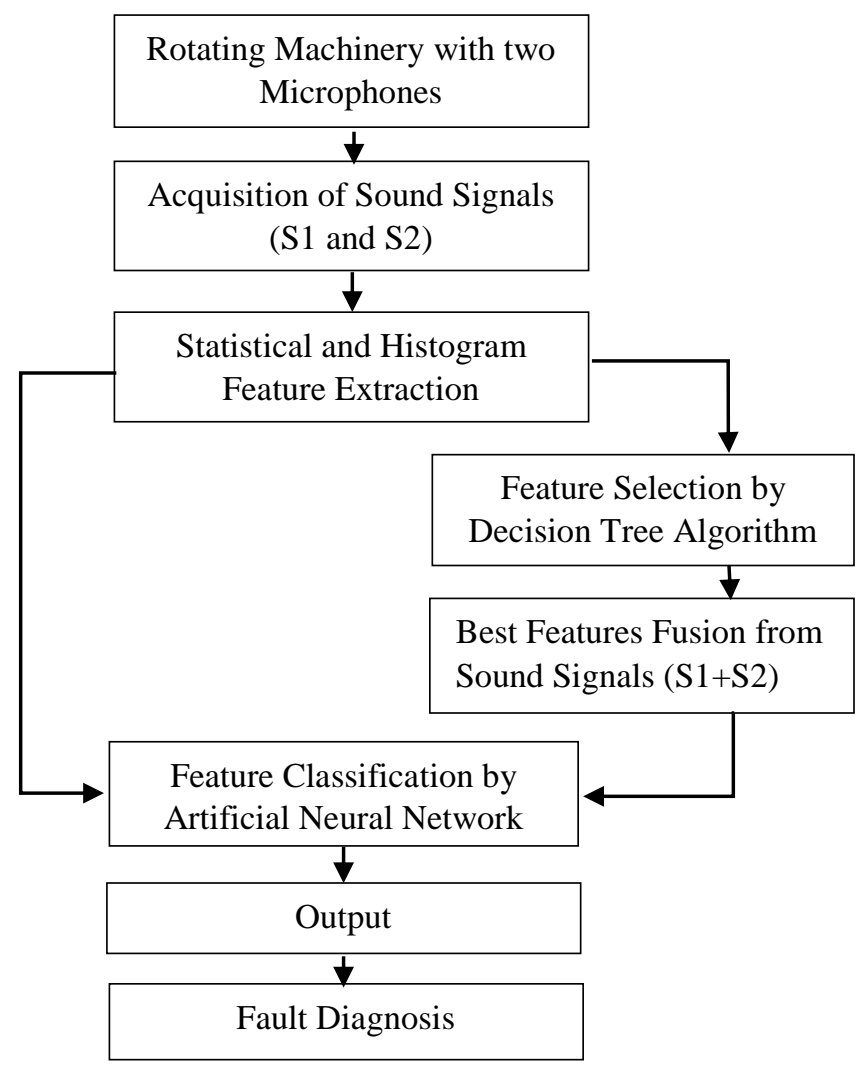

Figure 1. Flow Chart Representation of Fault Diagnosis Procedure.

This paper is organized as follows. Section 2 details about the setup and procedure of the experiment. Section 3 contains the statistical features and histogram features of data extraction from the sound signals. Section 4 discusses about the decision tree algorithm for data fusion. Section 5 details about the Artificial Neural Network classifier. Section 6 describes results and compares the classification accuracy of Statistical and histogram features. The last section presents the conclusions.

\section{EXPERIMENTAL ANALYSIS}

\subsection{EXPERIMENTAL SETUP}

Machinery Fault Simulator is used for studying the vibration and sound signals pattern by artificially creating faults in rotating machine elements. The setup consists of the variable DC motor (0.5 hp) with maximum speed of $3000 \mathrm{rpm}$, which is the driver. It is connected to shaft with the help of flexible coupling. The shaft is supported by means of two ball bearings. One bearing is placed near motor shaft is free from defect and other one has defects in it. A rotor having slots to provide eccentric loading in shaft is placed in between bearings. The basic requirement for acquisition of sound signals is that microphones connected to Analogue Digital Converter (ADC). Microphones are placed near the bearings to collect the sound signals. Microphone signals are send to analogue digital convertor, where signal conditioning and filtering of noises is done. Then the data is sent to the software for obtaining the time domain signal. The experimental setup is pictured in Figure 2.

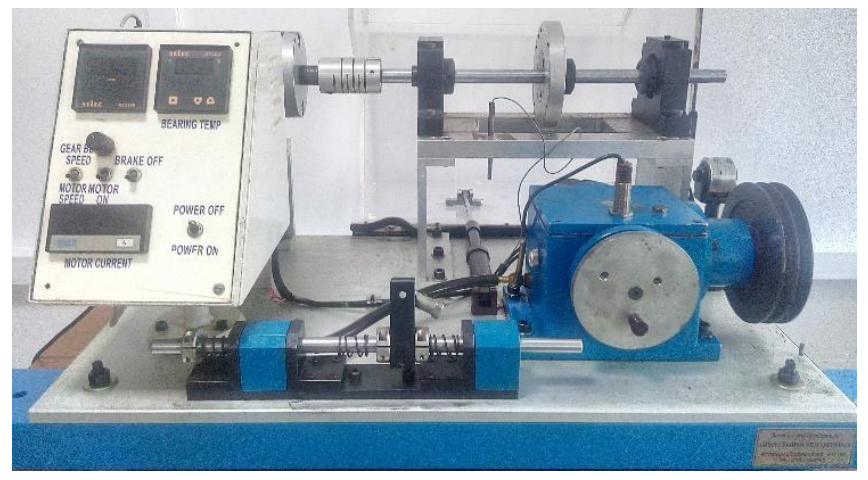

Figure 2. Experimental setup.

\subsection{Experimental Procedure}

In this experiment six different fault classes are used. They are Good Shaft with Good bearing (f1), Unbalanced Shaft with Good bearing (f2), Good Shaft with Outer Race Fault in bearing (ORFB) (f3), Unbalanced Shaft with Outer Race Fault in bearing (ORFB) (f4), Good Shaft with Inner Race Fault in bearing (IRFB) (f5), Unbalanced Shaft with Inner Race Fault in bearing (IRFB) (f6). Two types of bearings are used, Bearing near flexible coupling is good and other one has fault. The simulation is done for three different speeds of $400 \mathrm{rpm}, 600 \mathrm{rpm}$ and $800 \mathrm{rpm}$. The 8192 data sets per second is obtained for time of 100 seconds. The sound data (S1) for different fault classes at speed of $600 \mathrm{rpm}$ is shown in Figure 3. 


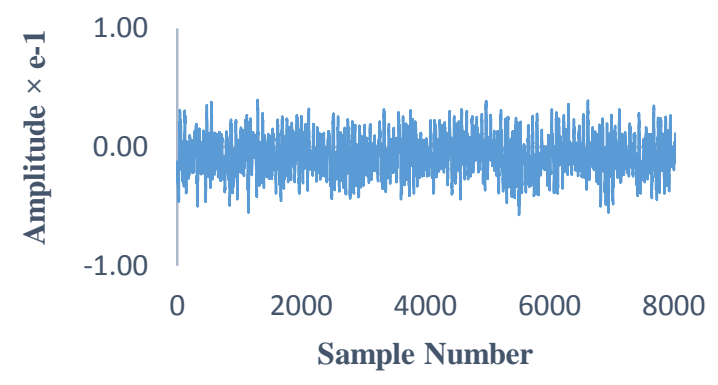

Good shaft with good bearing

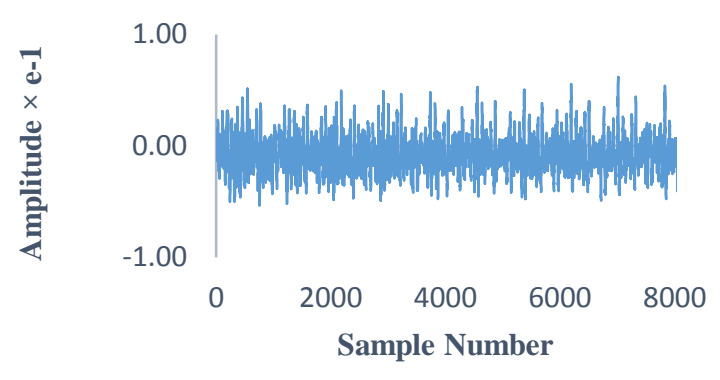

Unbalanced Shaft with good bearing

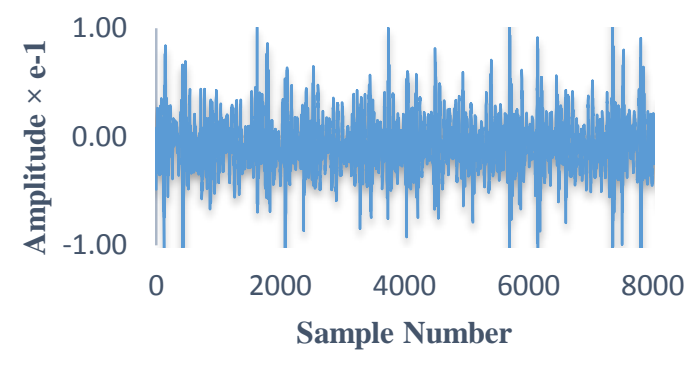

Good Shaft with IRFB

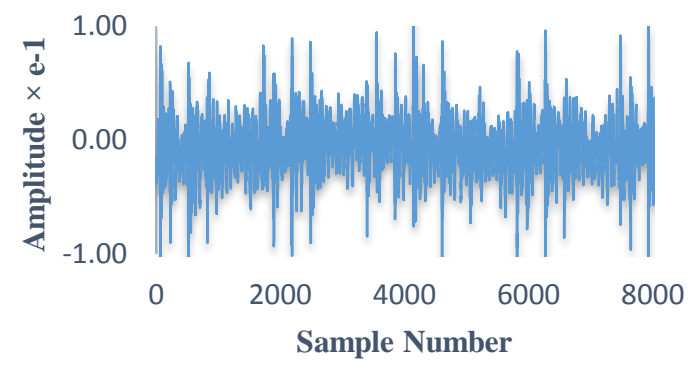

Unbalanced Shaft with IRFB

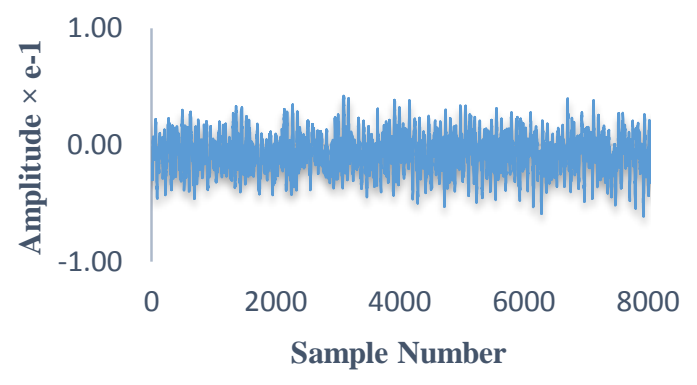

Good Shaft with ORFB

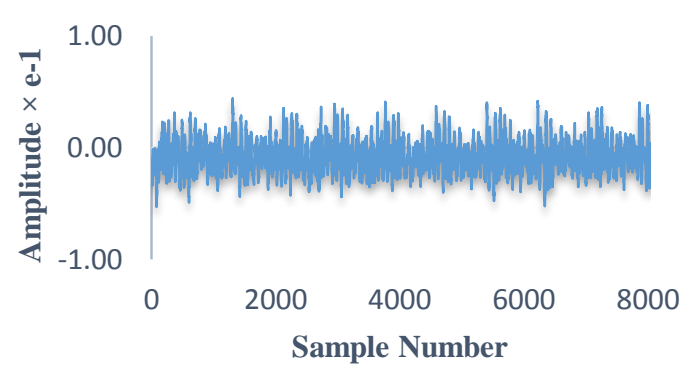

Unbalanced Shaft with ORFB

Figure 3. Time domain plots of Sound signals (S1) for different fault conditions at $600 \mathrm{rpm}$.

As it is seen from Figure 3. Time domain plots are used for identification of fault in a component with the help of amplitude of signals. The amplitudes peaks for both conditions of good shaft with IRFB and Unbalanced shaft with IRFB.

\section{FEATURE EXTRACTION}

Feature Extraction Processes are carried out to extract the information that each fault condition class contain. The Feature extraction processes enables to define machine's health status. The feature extraction is done through different features like statistical, histogram, wavelet transforms. Here Statistical and histogram features are carried out.

\subsection{Statistical Features}

Statistical analysis of sound signal gives different parameters. This method involves extraction of data with statistical methods. They are mean, standard deviation, sample variance, median, mode, sum, maximum and minimum (Vapnik, 1999; Saimurugan, Ramachandran, Sugumaran \& Sakthivel, 2011).

(a) Mean: It is the average value of sound signals. Mean is calculated by formula in Eq. (1) 


$$
\bar{x}=\frac{x_{1}+x_{2}+x_{3}+\cdots x_{n}}{n}
$$

(b) Standard Deviation: It is the measure of effective energy of a sound signals. The Standard deviation $\left(\mathrm{S}_{\mathrm{n}}\right)$ is calculated by formula in Eq. (2)

$$
S_{n}=\sqrt{\frac{1}{n} \sum_{i=1}^{n}\left(x_{i}-\bar{x}\right)^{2}}
$$

(c) Sample Variance: It is variance of sound signal points. Variance $\left(\mathrm{s}^{2}\right)$ is calculated by formula in Eq. (3)

$$
S^{2}=\frac{1}{n-1} \sum_{i=1}^{n}\left(x_{i}-\bar{x}\right)^{2}
$$

(d) Median: The sound data set values are set in order to find the middle data value.

(e) Mode: Mode is used to identify most recurring value in a Sound data set.

(f) Sum: It is the sum of all signal points in a given sound signals.

(g) Maximum: It refers to the crest signal value in sound signals.

(h) Minimum: It refers to the trough signal value in sound signals.

These features are extracted from sound signals at different speeds.

\subsection{HISTOGRAM FEATURES}

Histograms are plotted for the continuous set of data for the identification of distribution of the data. The data is first split to specified intervals known as bins. In fault diagnosis procedure, the width of the bin is made constant. In histograms, the area under the bar becomes important and not the amplitude of the signals. But bin range must accommodate the amplitudes of six different fault condition. From the area under the bar, the frequency of occurrence of signal data can be calculated. The samples shown below indicates different set of bearings and shaft fault condition. Selection of bin involves two conditions viz. 1. Bin range is used to plot histogram and it depends on the maximum and minimum value in the range. The minimum and maximum values of the different bearing and shaft conditions are the small and large values respectively of the bin range. 2. Bin width must be fixed and Amplitude range is chosen in a way to distinguish different fault conditions. Bin width is calculated from Figure 4. as (-0.4-(-0.6)) which is 0.2 (Sugumaran \& Ramachandran, 2011). The two conditions are amplitude range of the signal and width of the bin. Following are the histogram plots for each condition at $600 \mathrm{rpm}$ speed in Figure 4.

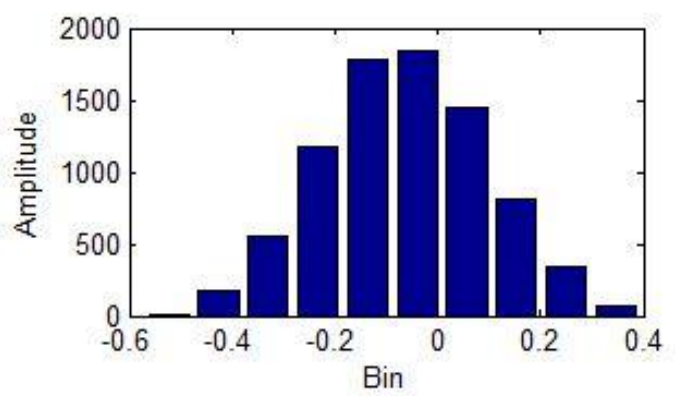

Good Bearing with Good Shaft

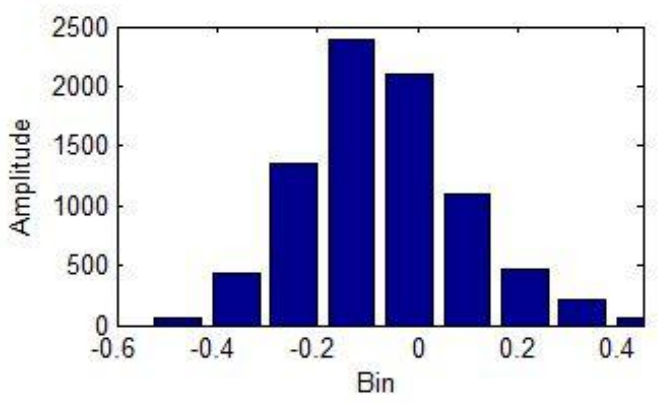

Good Bearing with Unbalanced Shaft

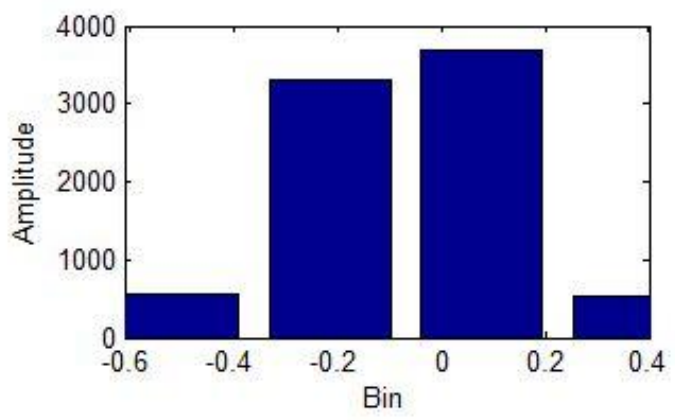

Good shaft with IRFB 


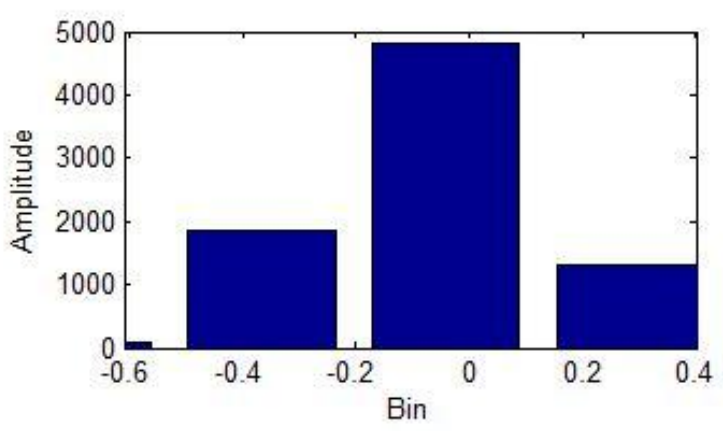

Unbalanced Shaft with IRFB

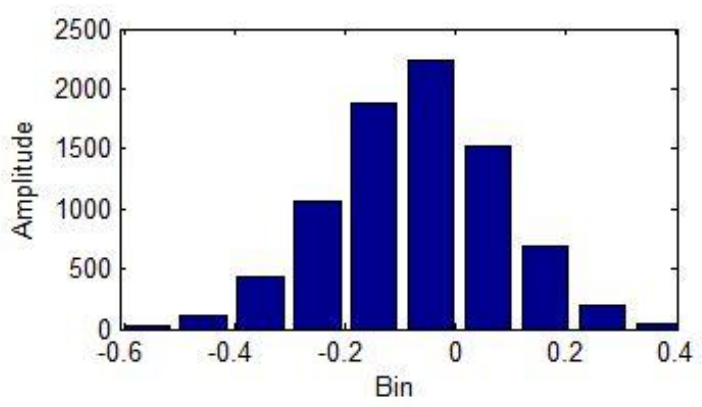

Good shaft with ORFB

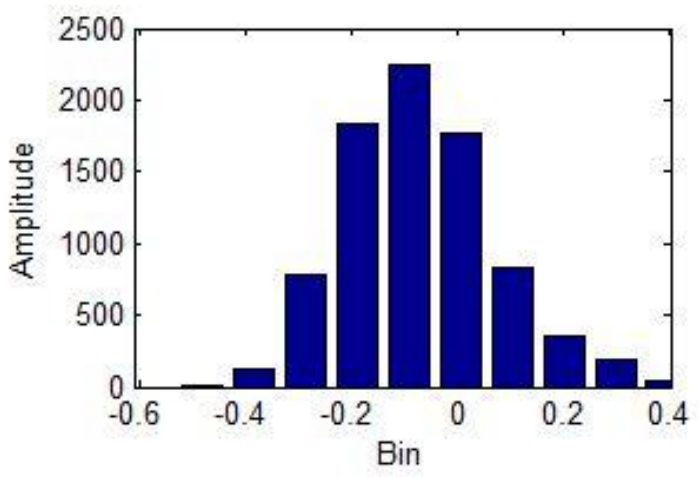

Unbalanced Shaft with ORFB

Figure 4. Histogram Plots of Sound signal (S1) for different fault classes at $600 \mathrm{rpm}$ speed

The above plots show the difference in amplitude range for the each fault condition. The selected features are given as inputs to decision tree.

\section{DeCision Tree Algorithm}

Decision tree is method of data interpretation in the form of a tree. This method has a root node, which separates to form the daughter nodes or leaf nodes. The final leaf node represents the probability of the outcome. It is arranged in such a fashion that root node forms primary attribute containing maximum information while the last leaf contains the least information.

C4.5 algorithm is the widely used algorithm for decision tree (Quinlan, 1993). This algorithm was developed after Induction Decision tree version 3 (ID3).C4.5 algorithm minimizes errors in comparison with ID3. C4.5 algorithm involves recursive method of classification of information. First, select the root node attribute $\mathrm{X}$, accuracy of classification depends only on how the root node is selected. In sample $E$, any attribute $X$ is selected and it is partitioned to daughter nodes, for instance $E_{1}, E_{2}, E_{3}, \ldots E_{n}$. Further, the daughter nodes for $\mathrm{E}$ is repeatedly partitioned until required information is obtained. This algorithm uses entropy information for calculation. Entropy is the measure of disorder by which pure nodes can be achieved.

Information entropy for set $\mathrm{E}$ is given by,

$\operatorname{Info}(E)=-\sum_{i=1}^{k}\left(\left(\frac{\operatorname{freq}\left(D_{\mathrm{i}}, E\right)}{|E|}\right) \times \log 2 \times\left(\frac{f r e q\left(D_{\mathrm{i}}, E\right)}{|E|}\right)\right)$

Here, $|E|$ is the total number of cases for training. $D_{i}$ is a type of class $(\mathrm{i}=1,2,3 \ldots \mathrm{n}) . \mathrm{K}$ is the number of classes and freq $\left(D_{i}, E\right)$ is the number of cases included in $D_{i}$.

Expected information value is calculated from formula,

$$
\operatorname{Info}_{\mathrm{x}}(E)=-\sum_{i=1}^{k}\left(\left(\frac{\left|E_{\mathrm{i}}\right|}{|E|}\right) \times \operatorname{Info}\left(E_{\mathrm{i}}\right)\right)
$$

Gain in information is given by,

$$
\operatorname{Gain}(\mathrm{X})=\operatorname{Info}(E)-\operatorname{In} f o_{\mathrm{X}}(E)
$$

The decision tree algorithm is applied to the statistical data features for different fault condition and different speeds and the result shown in Figure 5.

The tree used for this method is based on C4.5 algorithm. The data fusion is technique of combining or fusing the best features of signals. From Figure 5. it's clear that best features are sample variance, minimum, mean and median. Then the best features from microphone signal 2 are similarly extracted and the best features of both the signals are finally fused to form the fusion signal. The results of fused sound signals are compared with the individual signals for classification accuracy. 


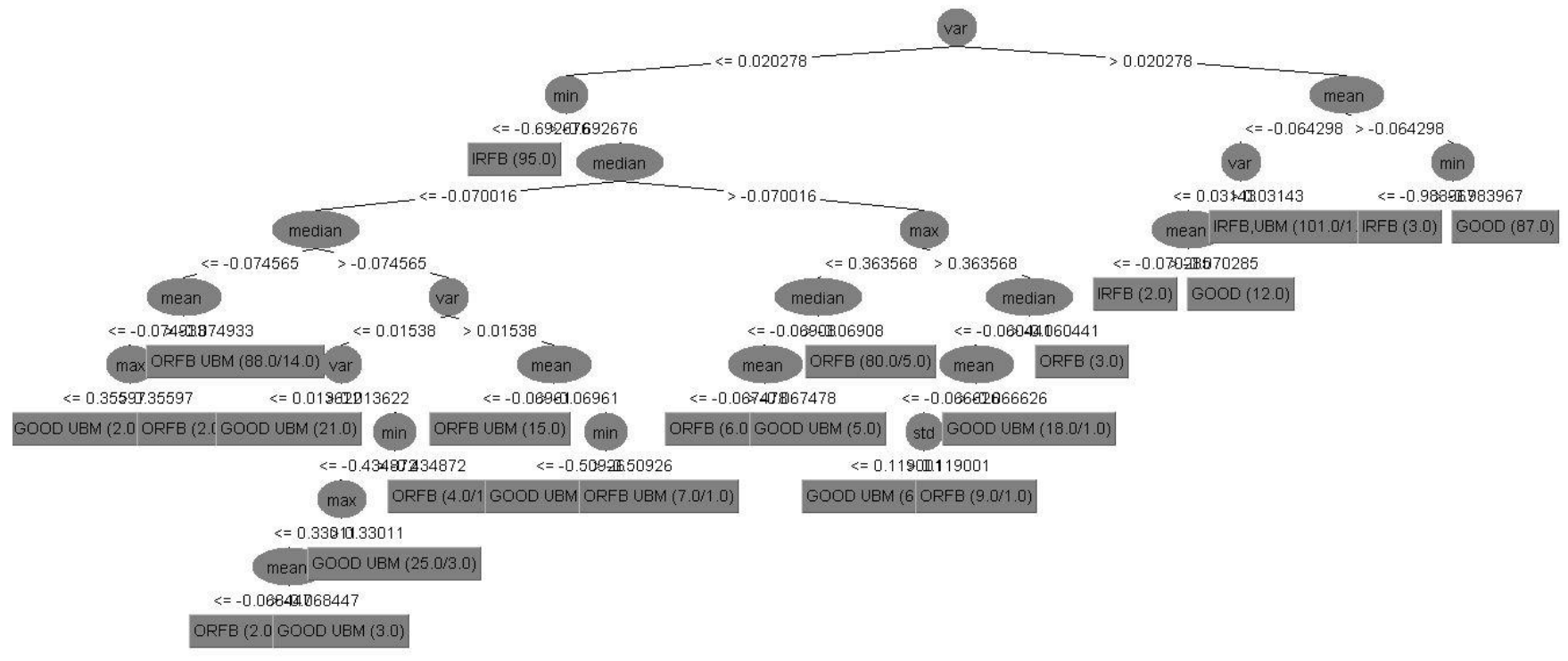

Figure 5. Decision Tree for sound signal (S1) from statistical feature at $400 \mathrm{rpm}$

\section{Artificial Neural Network}

Artificial Neural Network is a simulated model as that of working of a neural network of a human brain. This model works by receiving signals from ' $n$ ' different sources and then the signals travel through weighted pathways $\mathrm{W}_{\mathrm{i}}$ in order to activate internal activation energy $\mathrm{X}_{\mathrm{j}}$. This is subsequently transformed to generate the output signal function. The neural network model is capable of giving output even in noisy or bulk data.

This model is an analogy to the human brain. Neural network in human consists of dendrites in the form of tree like structure. The input signals are received through dendrites and output signals are carried away by axon. Communication between neurons takes place through the specialized points called synapses. The artificial neuron model is represented below in Figure 6.

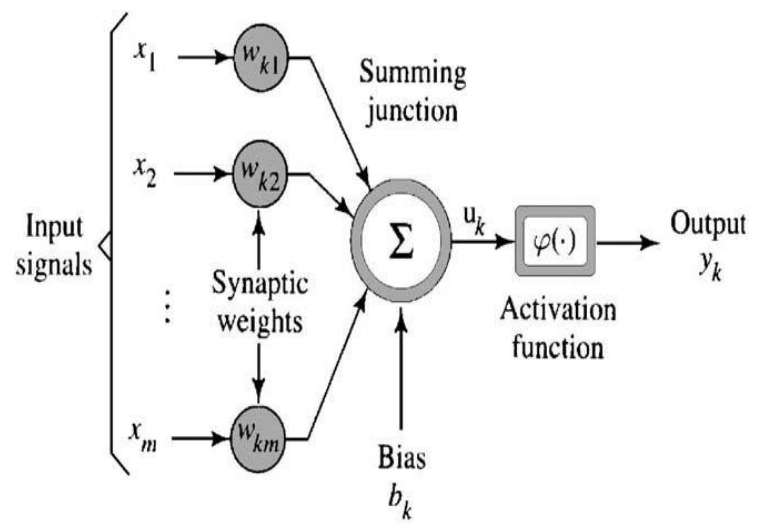

Figure 6. Non-linear model of neuron (Fonseca, Navaresse \& Moynihan, 2003)

Here $X_{1}, X_{2} \ldots X_{m}$ are the input signals. The input signals are multiplied by weighted function or bias $\left(b_{k}\right)$. Then output is obtained after passing through activation function. Neuron signal functions can be of type like binary threshold, linear, sigmoid, Gaussian etc. In this paper sigmoidal signal function is used.

The artificial neural networks forms an architecture to generate output function. Artificial Neural Network Architecture is shown in Figure7.

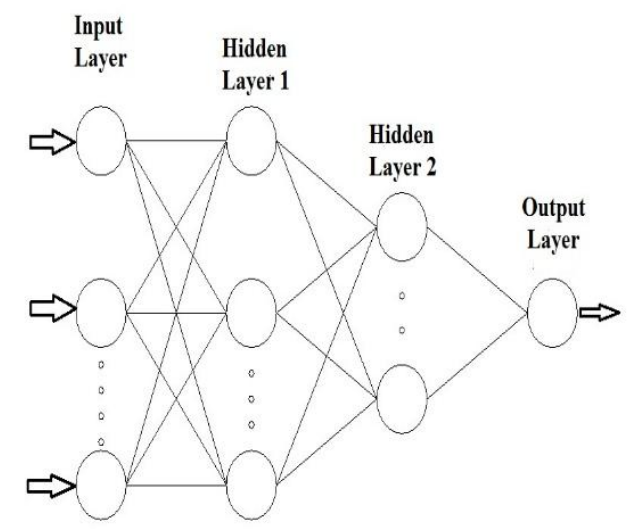

Figure 7. Artificial Neural Network Architecture 
This architecture has input layers and output layers with hidden layers as intermediate layers. The architecture is of two types' viz. Feed forward neural network and back propagation neural network. The difference is that back propagation neural network will have feedback. The hidden layer forms the intermediate data transfer neurons. In this paper, the widely used back propagation neural network is used to classify data sets.

A summary of the back propagation algorithm as follows (Caudill \& Butler, 1992)

1. The input to the hidden layer and output layer is given by,

$$
I_{i}=\sum_{j=1}^{n}\left(x_{i} w_{i j}+b_{k}\right)
$$

Here $\mathrm{x}$ is the output of the previous layer, $b_{k}$ is the bias function and $w$ is the weight function.

2. The output to the hidden layer and output layer is given by $y=f(I)$

$$
f(I)=\frac{1}{1+e^{-1}}
$$

Here $f(I)$ is a tan-sigmoid function.

3. In the training process, the change in weight function is given by delta-rule

$$
w_{i j}=L_{r} E f(I)+m \Delta w_{i j} \text { (previous) }
$$

Here $L_{r}$ is the learning rate, $\mathrm{E}$ is the error and $\mathrm{m}$ is the momentum constant. The range of both $L_{r}$ and $\mathrm{m}$ varies from 0 to 1.

4. The output layer contains some error and it is given by,

$$
E_{i}^{\text {output }}=y_{i}^{\text {desired }}-y_{i}^{\text {actual }}
$$

5. The error in the hidden layer is obtained from the output layer by back propagation and then it is multiplied by the derivative of the hidden layer neuron's activity.

$$
E_{i}^{\text {hidden }}=\frac{d f I_{i}^{\text {hidden }}}{d I} \sum_{j=1}^{n}\left(w_{i j} E_{i}^{\text {output }}\right)
$$

The parameters for tuning in neural networks are learning rate, momentum and number of neurons in the hidden layer. The value of learning constant or learning rate is chosen as 0.3 and the momentum constant value is taken as 0.2 based on trial and error method for increasing the classification accuracy. There is no accurate formula to find the number of neurons in the hidden layer. The number of neurons in the hidden layer $\left(N_{H}\right)$ is evaluated by the rule of thumb formula

$$
N_{H}=\frac{N_{V}+N_{c}}{2}+\sqrt{N_{D}}
$$

Here $N_{V}$ is the number of input variables, $N_{C}$ is the number of output classes and $\mathrm{N}_{\mathrm{D}}$ is the number of data samples.
The number of hidden neurons is varied \pm 5 from the value obtained in Eq. (12) and the best suitable number of hidden neuron is determined based on classification accuracy (Chow, Lee \& Li, 2012). From the Eq. (12), the number of

\begin{tabular}{|c|c|c|c|c|c|c|}
\hline$N_{H}$ & 17 & 18 & 19 & 20 & 21 & 22 \\
\hline $\begin{array}{l}\text { Accuracy } \\
\%\end{array}$ & 86.2 & 87.3 & 86.5 & 86.2 & 86.3 & 87.6 \\
\hline$N_{H}$ & 16 & 15 & 14 & 13 & 12 & \\
\hline $\begin{array}{l}\text { Accuracy } \\
\%\end{array}$ & 87.2 & 86.5 & 87.3 & 86.2 & 86.5 & \\
\hline
\end{tabular}
hidden neuron for statistical and histogram features are found to be 17 and 18 respectively. Table 1 and Table 2 shows the range of possible $N_{H}$ values and their prediction accuracy for statistical features of the sound signals $\mathrm{S} 1$ and

\begin{tabular}{|c|c|c|c|c|c|c|}
\hline$N_{H}$ & 17 & 18 & 19 & 20 & 21 & 22 \\
\hline $\begin{array}{l}\text { Accuracy } \\
\%\end{array}$ & 73.2 & 73.6 & 74.6 & 73.9 & 74 & 74.3 \\
\hline$N_{H}$ & 16 & 15 & 14 & 13 & 12 & \\
\hline $\begin{array}{l}\text { Accuracy } \\
\%\end{array}$ & 74.1 & 72.6 & 73.8 & 73.3 & 73.5 & \\
\hline
\end{tabular}
$\mathrm{S} 2$ at the speed of $400 \mathrm{rpm}$ respectively.

Table 1. The range of possible $N_{H}$ values and their prediction accuracy for statistical features of the sound signal S1 at the speed of $400 \mathrm{rpm}$.

Table 2. The range of possible $N_{H}$ values and their prediction accuracy for statistical features of the sound signal S2 at the speed of $400 \mathrm{rpm}$.

From Table 1 and Table 2, it is clear that almost maximum

\begin{tabular}{|c|c|c|c|c|c|c|}
\hline$N_{H}$ & 18 & 19 & 20 & 21 & 22 & 23 \\
\hline $\begin{array}{l}\text { Accuracy } \\
\%\end{array}$ & 78.2 & 78.6 & 77.5 & 76.8 & 77.6 & 78.5 \\
\hline$N_{H}$ & 17 & 16 & 15 & 14 & 13 & \\
\hline $\begin{array}{l}\text { Accuracy } \\
\%\end{array}$ & 78.2 & 79.5 & 78 & 76.6 & 77.2 & \\
\hline
\end{tabular}
accuracy is observed at maximum hidden neuron number in that range i.e. at $N_{H}=22$ for statistical features. Table 3 and Table 4 shows the range of possible $N_{H}$ values and their prediction accuracy for histogram features of the sound

\begin{tabular}{|c|c|c|c|c|c|c|}
\hline$N_{H}$ & 18 & 19 & 20 & 21 & 22 & 23 \\
\hline $\begin{array}{l}\text { Accuracy } \\
\%\end{array}$ & 75 & 76.5 & 77.6 & 74.3 & 76.5 & 77.6 \\
\hline$N_{H}$ & 17 & 16 & 15 & 14 & 13 & \\
\hline $\begin{array}{l}\text { Accuracy } \\
\%\end{array}$ & 74.5 & 75.3 & 74.5 & 73.3 & 76 & \\
\hline
\end{tabular}
signals S1 and S2 at the speed of $400 \mathrm{rpm}$ respectively.

Table 3. The range of possible $N_{H}$ values and their prediction accuracy for histogram features of the sound signal S1 at the speed of $400 \mathrm{rpm}$. 
Table 4. The range of possible $N_{H}$ values and their prediction accuracy for histogram features of the sound signal S2 at the speed of $400 \mathrm{rpm}$.

From Table 3 and Table 4, it is clear that almost maximum accuracy is observed at maximum hidden neuron number in that range i.e. at $N_{H}=23$ for histogram features. Hence the number of hidden neurons $N_{H}$ for statistical and histogram features are 22 and 23 respectively can be chosen for further classification process.

The classifier neural network involves phases like training and testing. Training or learning phase involves prediction of class labels to the input data based on bias function and the resulting output is matched with desired output. If there is an error, then bias functions are suitably adjusted to increase the efficiency. The developed trained model has to be validated and verified by testing process. Various techniques are available to split the datasets for training and testing the machine learning algorithm. The K-fold cross validation is the best method in order to avoid "overfitting" and "underfitting" in the trained model (Hertz, Krogh \& Palmer, 1992; Salzberg, 1997).In K-fold cross validation, the available data samples are divided into $\mathrm{K}$ disjoint subsets where $(K-1)$ subsets are used as a training data and remaining one subset is used as a validation data for testing the model. This process is repeated for $\mathrm{K}$ times and the average of the $\mathrm{K}$ results produce a model estimation. In this research work, $\mathrm{K}$-fold cross validation method $(\mathrm{K}=10)$ is used to evaluate the ANN model.

\section{RESULTS AND DISCUSSION}

In this classification, back propagation neural network is used with two layers: Hidden layer is used with tan-sigmoid function and an output layer. Decision Tree is used to extract best four features from which fusion data sets are made and given as input to classifier. Testing is done through 10-fold cross validation technique.

The Classification accuracy is obtained in the form of confusion matrix from Artificial Neural Network classifier. Confusion matrix for statistical fusion signal at $600 \mathrm{rpm}$ is shown in Table 5.

\begin{tabular}{|l|l|l|l|l|l|l|}
\hline FC $^{*}$ & f1 & f2 & f3 & f4 & f5 & f6 \\
\hline f1 & 100 & 0 & 0 & 0 & 0 & 0 \\
\hline f2 & 0 & 100 & 0 & 0 & 0 & 0 \\
\hline f3 & 0 & 0 & 97 & 0 & 1 & 2 \\
\hline f4 & 0 & 0 & 0 & 100 & 0 & 0 \\
\hline
\end{tabular}

\begin{tabular}{|l|l|l|l|l|l|l|}
\hline f5 & 0 & 1 & 0 & 0 & 95 & 4 \\
\hline f6 & 0 & 11 & 0 & 0 & 5 & 84 \\
\hline
\end{tabular}

*FC - Fault Classes

Table 5. Confusion matrix for fusion signals from statistical method at 600 rpm using Artificial Neural Network classifier.

From the above matrix, the diagonal elements of matrix shows accurately classified instances. In the first row Good shaft and good bearing (f1) has the classification accuracy of $100 \%$. Few data (f3) of good shaft with outer race fault bearing (f3) is misclassified as (f5) and (f6). The major misclassification occurs between (f6) (f2) and (f6) (f5). The accuracy of matrix is found to be $94.5 \%$

The classification accuracy of Statistical and Histogram features at different speeds are shown in Table 6 and Table 7 respectively.

\begin{tabular}{|c|c|c|c|}
\hline $\begin{array}{c}\text { Speed } \\
(\mathrm{rpm})\end{array}$ & \multicolumn{3}{|c|}{ Classification Accuracy (\%) } \\
\hline & $\begin{array}{c}\text { Mic 1 } \\
\text { (Sound) }\end{array}$ & $\begin{array}{c}\text { Mic 2 } \\
\text { (Sound) }\end{array}$ & Fusion \\
\hline 400 & 87.6 & 74.3 & 89.3 \\
\hline 600 & 89.5 & 84.2 & 94.5 \\
\hline 800 & 75.5 & 59.1 & 77.2 \\
\hline
\end{tabular}

Table 6. Classification accuracy of Statistical features using Artificial Neural Network classifier.

\begin{tabular}{|c|c|c|c|}
\hline $\begin{array}{c}\text { Speed } \\
(\mathrm{rpm})\end{array}$ & \multicolumn{3}{|c|}{ Classification Accuracy (\%) } \\
\hline & $\begin{array}{c}\text { Mic 1 } \\
\text { (Sound) }\end{array}$ & $\begin{array}{c}\text { Mic 2 } \\
\text { (Sound) }\end{array}$ & Fusion \\
\hline 400 & 78.5 & 77.6 & 81.3 \\
\hline 600 & 74.2 & 74 & 75.3 \\
\hline 800 & 58.7 & 58.5 & 64.4 \\
\hline
\end{tabular}

Table 7. Classification accuracy of Histogram features using Artificial Neural Network classifier.

Table 6 and Table 7 shows that statistical features gives better classifier result compared to histogram features. Classification accuracy of fusion signals are high compared to individual sound signals.

Hence by using fusion data fault diagnosis can be improved. Automated fault diagnosis can be done using fusion of sound signals. 


\section{CONCLUSION}

Condition monitoring of rotating machinery is evolving area in research. Fault diagnosis of bearings and shafts mostly uses vibration signals, here sound signals are used. The Decision tree (C4.5 Algorithm) is used to select features, from which fusion signals are formed. The feature classification is done with Artificial Neural Network and the classification accuracy is found out from confusion matrix. The classification accuracy of statistical and histogram features are compared and from the results, Statistical features are found to be a good candidate for fault diagnosis using sound signals. Fusion data signals have good classification accuracy compared to individual accuracy of signals.

\section{REFERENCES}

Vyas, N.S., \& Satishkumar, D. (2001) Artificial neural network design for fault identification in a rotor-bearing

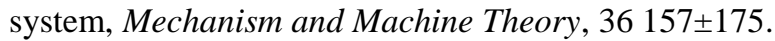

Randall, Robert Bond. (2011), Vibration-based condition monitoring - Industrial, aerospace and automotive applications. UK: John Wiley and Sons, Ltd., Publication.

Samanta, B., \& Al-Balushi, K.R. (2003). Artificial neural network based fault diagnostics of rolling element bearings using time domain features, Mechanical Systems and Signal Processing 17 (2), 317-328.

Quinlan, J. R. (1993). C4.5: Programs for machine learning. San Mateo, CA: Morgan, Kaufmann.

Sugumaran,V., \& Ramachandran,K.I. (2011). Fault diagnosis of roller bearing using fuzzy classifier and histogram features with focus on automatic rule learning, Expert Systems with Applications $38,4901-4907$.

Samadani, M., Kitio Kwuimy, C.A., \& Nataraj, C. (2015). Model-based fault diagnostics of nonlinear systems using the features of the phase space response. Communications in Nonlinear Science and Numerical Simulation 20.2: 583-593.

Zhiqiang Chen, Chuan Li \& René Vinicio Sánchez. (2015). Multi-layer neural network with deep belief network for gearbox fault diagnosis. Journal of Vibroengineering. Vol. 17, Issue 5, p. 2379-2392.

Chuan Li, Ming Liang, Tianyang Wang. (2015). Criterion fusion for spectral segmentation and its application to optimal demodulation of bearing vibration signals, Mechanical Systems and Signal Processing 64-65, 132148.

Heng, R. B. W., \& Nor, M. J. M. (1998). Statistical Analysis of Sound and Vibration Signals for Monitoring Rolling Element Bearing Condition. Applied Acoustics, Vol. 53, No. 1-3, pp. 21 1-226.

Alaa Abdulhady Jaber \& Robert Bicker. (2016). Fault Diagnosis of Industrial Robot Bearings Based on Discrete Wavelet Transform and Artificial Neural
Network. International Journal of Prognostics and Health Management, ISSN 2153-2648, 2016017.

Amarnath, M., Sugumaran, V., Hemantha Kumar. (2013). Exploiting sound signals for fault diagnosis of bearings using decision tree. Measurement 46, 1250-1256.

Vapnik, V.N., (1999). An overview of statistical learning theory. IEEE transactions on Neural Networks 10, 9881000.

Fonseca, D.J., Navaresse, D.O., \& Moynihan, G.P. (2003). Simulation Meta modeling through artificial neural networks. Engineering Applications of Artificial Intelligence 16, 177-183.

Saimurugan, M., Ramachandran, K.I., Sugumaran, V., \& Sakthivel, N.R. (2011). Multi- component fault diagnosis of rotational mechanical system based on decision tree and support vector machine. Expert Systems with Applications 38, 3819-3826.

Smalley, A. J., Baldwin, R. M., Mauney, D. A., \& Millwater, H. R. (1996). Towards risk based criteria for rotor vibration. International proceedings of the institute of mechanical engineers-Vibrations in rotating machinery (pp. 517-527).

Kankar, P.K., Sharma Satish, C., \& Harsha, S.P. (2011). Fault diagnosis of ball bearings using machine learning methods. Expert Systems with Applications 38, 18761886.

Widodo, A., \& Yang, B.S. (2007). Review on support vector machine in machine condition monitoring and fault diagnosis. Mechanical Systems and Signal Processing, 21, 2560-2574.

Yuan, S., \& Chu, F. (2007). Fault diagnosis based on support vector machines with parameter optimization by artificial immunization algorithm. Mechanical Systems and Signal Processing, 21, 1318-1330.

Meirovitch, Leonard. (1986). Elements of Vibration analysis. USA: McGraw-Hill Publication.

Caudill, M., \& Butler, C. (1992). Understanding Neural Networks: Computer Explorations, MIT Press, Cambridge, MA.

Salzberg, S.L. (1997). On comparing classifiers: Pitfalls to avoid and a recommended approach. Data mining and knowledge discovery, 1(3), pp.317-328.

Hertz, J., Krogh \& Palmer, R.G. (1991). Introduction to the Theory of Neural Computation. Addison Wesley. California.

Chow, S.K.H., Lee, E.W.M., Li, D.H.W. (2012). Short-term prediction of photovoltaic energy generation by intelligent approach, Energy and Buildings 55, 660-667.

Xin Lei \& Peter A. Sandborn. (2016). PHM-Based Wind Turbine Maintenance Optimization Using Real Options. International Journal of Prognostics and Health Management, ISSN 2153-2648, 2016008. 


\section{BIOGRAPHIES}

Saimurugan $\mathbf{M}$ received his B.E degree in Mechanical Engineering from Kongu Engineering College, Bharathiar University, Coimbatore, in 1998. He received his M. E. degree in Computer Aided Design from Government College of Engineering, Periyar University, Salem, in 2000. He received his Ph.D. from Amrita School of Engineering, Amrita Vishwa Vidyapeetham, Coimbatore, in 2013. He currently serves as Assistant Professor at department of Mechanical Engineering, School of Engineering, Coimbatore Campus. His areas of research include Vibration Analysis, Machine Learning and Machine Condition Monitoring.

Nithesh $\mathbf{R}$ currently pursuing his B. Tech degree in Mechanical Engineering from Amrita School of Engineering, Amrita Vishwa Vidyapeetham, Coimbatore. His areas of research include Machine Condition Monitoring. 